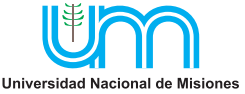

Vol. $2 \mathrm{~N}^{\circ}$ 2, julio-diciembre 2020, pag. 90-101

ISSN 2618-5520 on line

DOI - j.masingenio.2021.02.02.008

\title{
Transición hacia la formación por competencias: un análisis en la Facultad de Ingeniería - UNMDP
}

\author{
Onaine A.E.*, Artigas, M.V., Santille L.S. \\ Facultad de Ingeniería, Universidad Nacional de Mar del Plata (UNMdP), Av. Juan B. Justo 4302, Mar del Plata, \\ Buenos Aires, Argentina. \\ e-mail: aeonaine@fi.mdp.edu.ar;mvartigas@hotmail.com; lusantille@gmail.com
}

\begin{abstract}
Resumen
El objetivo de este trabajo es presentar un análisis sobre cómo se transita la reformulación de los planes de estudios incorporando competencias en particular, haciendo foco en las percepciones de docentes de la Facultad de Ingeniería de la Universidad Nacional de Mar del Plata (FI-UNMdP). El estudio releva las voces de docentes en cuanto a sus prácticas y perspectivas para poder describir y explicar este proceso. Desde el año 2018 se han iniciado estrategias tendientes al cambio en los procesos de enseñanza-aprendizaje hacia un modelo por competencias. La metodología es cualitativa, con entrevistas semi-estructuradas a docentes exclusivos y parciales, luego se utiliza el análisis del contenido del discurso en tres niveles de análisis: informacional, estructural y social-hermenéutico. A partir de la presente indagación se registran resultados en cuanto a las percepciones expresadas sobre sus prácticas docentes; en relación a sus ideas en cuanto a la modificación profunda que el cambio supone, debido a que implica transformar el modo de pensar y enseñar; y sus impresiones con respecto a la transición. Finalmente, se espera profundizar esta línea de investigación con otros estudios para determinar el impacto en los docentes de la transición y poder compararlo con acciones concretas del diseño curricular y prácticas docentes.
\end{abstract}

Palabras Clave - Competencias, Transición, Docentes, Análisis Discursivo, Percepciones, Cambio educativo

\begin{abstract}
The objective of this work is to present an analysis on how the reformulation of the curricula is carried out, incorporating competencies in particular, focusing on the perceptions of Professors from Facultad de Ingeniería Universidad Nacional de Mar del Plata (FI-UNMdP). The study surveys the voices of teachers in terms of their practices and perspectives in order to describe and explain this process. Since 2018, strategies have been initiated to change the teaching-learning processes towards a model by competencies. The methodology is qualitative, with semistructured interviews to full-time and part-time professors. Then, the analysis of the content of the discourse is used at three levels of analysis: informational, structural and social-hermeneutical. From the current investigation, results are presented regarding the perceptions of their teaching practices; in relation to their ideas regarding the profound modification that change implies, because it implies transforming the way of thinking and teaching; and their impressions regarding this transition. Finally, it is expected to deepen this line of research with other studies to determine the impact on professors of the transition and to be able to compare it with concrete actions of the curriculum design and teaching practices..
\end{abstract}

Keywords - Competences, Transition, Teachers, Discursive Analysis, Perception, Educational change 


\section{Introducción}

Para contextualizar, el presente trabajo se enmarca en un proyecto de investigación mayor cuyo objetivo es: conocer, describir y explicar los procesos intervinientes en la transición de un modelo de enseñanza-aprendizaje tradicional a un modelo por competencias centrado en el estudiante en la FI-UNMdP. Con ese marco y para este artículo, se focaliza sobre las ideas, percepciones y apreciaciones de un grupo de docentes sobre sus propias prácticas y cómo transitan ellos este cambio; en cuanto a la incorporación del modelo de formación por competencias en los planes de estudios y en su tarea docente.

Se configura el marco teórico de referencia específico tomando como eje central las conceptualizaciones sobre la formación por competencias. Asimismo es importante explicitar que si bien, la noción de percepciones tiene varias acepciones, se toman las definiciones de Carterette y Friedman [1]. Las explican como el resultado de un procesamiento de información que consta de estimulaciones a receptores en condiciones que en cada caso se deben parcialmente a la propia actividad del sujeto. Por lo tanto es una parte sustancial de la conciencia. Moya, citado en [2], explicita que lo que parece evidente es que en las percepciones intervienen los sentimientos, pensamientos y conductas respecto a las personas o sucesos y estarán mediatizados por el tipo de causa a la que se atribuya su conducta. Desde este aspecto, es que se considera tan importante entender cómo transitan los docentes del estudio el cambio, sus ideas, sus explicaciones, sus valoraciones dado que desde estas construcciones, sus percepciones se analizan. Estas visiones de docentes permiten comprender y describir la etapa inicial de dicho proceso de transición a un modelo de formación por competencias.

El contexto en el que se sitúa este estudio es la FI-UNMdP en la cual desde el año 2018, se trabaja institucionalmente en la revisión de los planes de estudios, con motivo de rever contenidos, metodologías y estar alineados con lo solicitado en relación a las futuras acreditaciones.

Los cambios en relación a la adecuación de los nuevos estándares vinculados a la formación por competencias, reflejados en varios documentos, los más recientes: la publicación del Libro Rojo del Consejo Federal de Decanos de Ingeniería (CONFEDI) y el Documento de Recomendaciones del Consejo Universitario, publicado en noviembre de 2019. Ambos ponen valor en la práctica docente como un modo de vehiculizar a los estudiantes de ingeniería con el desarrollo de saberes. En cuanto a conocimientos, al desarrollo de actitudes y al comportamiento. Entonces, se focaliza a través de entrevistas cómo vivencia el docente el proceso de cambio en relación enseñanza-aprendizaje basado en un modelo por competencias.

El objetivo es evidenciar las percepciones de un grupo de docentes, sus modos de pensar, sus prácticas y sus visiones con respecto al cambio y al proceso de transición de un modelo a otro.

Se plantea un estudio cualitativo circunscrito a la FI-UNMdP que utiliza entrevistas semiestructuradas, las cuales son realizadas a un grupo de docentes de la institución en cuestión. La muestra es intencional, no probabilística, esta selección contempla que pertenezcan a diferentes departamentos, con dedicaciones parciales y exclusivas. Entonces, con ellos se indaga el impacto en su propia vivencia, en sus prácticas docentes derivada de esta etapa inicial del proceso de cambio.

El CONFEDI define competencia como "la capacidad de articular eficazmente un conjunto de esquemas (estructuras mentales) y valores, permitiendo movilizar (poner a disposición) distintos saberes, en un determinado contexto con el fin de resolver situaciones profesionales" [3]. Otros autores entienden que las competencias son derivadas de procesos individuales tales como Klemp/Aguilar Joyas en [4] al considerar "las competencias como características del individuo relacionadas con el desempeño superior". Así como también en el proyecto Tuning las competencias "representan una combinación dinámica de las capacidades cognitivas y 
metacognitivas, de conocimiento y entendimiento, interpersonales, intelectuales y prácticas, así como de los valores éticos" [5].

Algunos autores contextualizan a las competencias en situación, tal los casos de: De Ketele en [4], que las define como "conjunto ordenado de capacidades (actividades) que son ejercidas sobre contenidos en una categoría dada de situaciones para resolver problemas que se presentan"; Perrenoud como "capacidad de actuar de manera eficaz en un tipo definido de situación, capacidad que se apoya en conocimientos, pero no se reduce a ellos. Para enfrentar una situación de la mejor manera posible, generalmente debemos hacer uso y asociar varios recursos cognitivos complementarios, entre los cuales se encuentran los conocimientos" [6]; Roegiers señala que es "la posibilidad, para un individuo, de movilizar, de manera interiorizada, un conjunto integrado de recursos con miras a resolver una familia de situaciones-problemas" [4]; y finalmente, Tobón como "procesos complejos de desempeño con idoneidad en un determinado contexto, con responsabilidad" [7].

Referentes nacionales como Kowalski, Erck y Henrriquez postulan que, en relación a la diferenciación de términos, hay que considerar que las aparentes inconsistencias radican en modelos educativos sustantivamente diferentes. Por tanto, es conveniente estar alertas para no quedar entrampados y terminar sosteniendo, por ejemplo, que educación basada en competencias es sinónimo de educación basada en resultados. Sin embargo, los autores acuerdan que las competencias aluden a integración de saberes, conductas - acciones y actitudes [8]. En el caso de Cukierman, la formación por competencias implica que el aprendizaje debe ser centrado en el estudiante (ACE) y que el profesor es el guía o "facilitador" ya que, desde su conocimiento y experticia, tanto de su rol docente como de la disciplina de que se trate, configura las estrategias y acciones necesarias para que sea el estudiante el que construya el conocimiento [9].

En relación al tema estudiado, se podría destacar un estudio sobre las percepciones de docentes sobre su formación en investigación realizado con categorías de análisis a priori definidas, utilizando el análisis discursivo como metodología [10]. Por otra parte, existen estudios que ponen el foco en las percepciones de docentes en relación al modelo de formación por competencias, indagando al docente visto como un experto quien tiene los conocimientos, motiva y gestiona los recursos pedagógicos para guiar el aprendizaje de sus estudiantes [11].

El análisis de práctica es un método de reflexión evolutivo [12] sobre el ejercicio de actividades, en este caso de docentes, que contribuye a la toma de conciencia de lo realizado y al mejoramiento de la capacidad de enseñar sobre la base de una combinación dinámica de atributos [13]. El método permite el razonamiento crítico de lo realizado y propicia la generación de los cambios de mentalidad que sean pertinentes a cada quien, mediante el uso de la reflexión sobre la propia práctica [13].

Se supone, en consecuencia, que para que una persona cambie su práctica, es preciso que el nuevo sistema de creencias multidimensional y complejo de valores domine su percepción individual de la realidad en la cual la persona se desempeña [15]. Que el saber teórico previo mute en una nueva acción [16] de modo que este cambio modifique las prácticas habituales e incorpore aptitudes, idoneidad y pericia [17]. Todas en conciencia y dominio, generando nuevos modelos mentales para que la práctica nueva e innovadora sea validada por los pares y finalmente sea asumida como la nueva regla que regulará y validará el nuevo saber [18].

En relación a las percepciones de docentes con respecto a competencias, el concepto de competencias es definido como holístico, polisémico y sistémico identifica genéricas, transversales, específicas, instrumentales y sistémicas. Desde esta perspectiva, el profesor es guía, facilitador para ayudar a los estudiantes a construir su propio conocimiento" [19]. 
Para sintetizar se podría decir que el concepto de competencia está focalizado en las capacidades y/o habilidades, conocimientos, destrezas, saber, hacer y saber hacer del ser humano que le permiten desempeñarse en el entorno donde se encuentre y de esta manera desenvolverse ante cualquier eventualidad o adversidad de los contextos [20].

Por último, con este trabajo se espera profundizar esta línea de investigación y resultados preliminares con otros estudios que permitan poder determinar el impacto en los docentes de la transición y poder compararlo con acciones concretas del diseño curricular y prácticas docentes.

\section{Desarrollo}

En una primera etapa se ha trabajado en profundizar el estudio del arte sobre el tema, delimitando un marco teórico referencial, que da un marco al trabajo experimental, en específico, se indaga sobre las ideas, percepciones y apreciaciones de un grupo de docentes sobre sus propias prácticas y cómo transitan ellos este cambio; en cuanto a la incorporación del modelo de formación por competencias en los planes de estudios y en su tarea docente.

Entonces, la investigación se inicia con una fase exploratoria de carácter cualitativo, cuyo objeto fue indagar los usos y prácticas sociales de los docentes con dedicaciones parciales y exclusivas, en relación al cambio de modelo de formación por competencias y sus prácticas. A partir de entrevistas a las personas que fueron consideradas informantes clave, se logra una producción discursiva, que involucra aspectos comunes y otros diferenciadores, también se tuvieron en cuenta aspectos sociodemográficos y psicolaborales sugeridos en la literatura [21].

\subsection{Metodología}

Se utilizan entrevistas semi-estructuradas como una metodología de recolección de datos de carácter cuantilativo de acuerdo a Glaser \& Strauss (1999) en [21]. Las mismas se nutren de los informantes clave, nuestros entrevistados, que posibilitan profundizar ciertos aspectos investigados. A través de esta herramienta, en este estadio exploratorio, se indagan las percepciones, los usos y prácticas sociales que los participantes del estudio atribuyen al proceso de cambio y transformación al nuevo modelo. Como elemento facilitador, se diseña un cuestionario con preguntas guía abiertas.

Para las entrevistas se establecieron las siguientes preguntas guía:

$\alpha$ ¿Estás en conocimiento de que se está iniciando un proceso de actualización de los planes de estudio de nuestra Facultad?

$\alpha \quad$ ¿Sabías que esa actualización responde a la reformulación en base a un modelo de enseñanza por competencias centrado en el estudiante?

$\alpha \quad$ ¿Leíste sobre los nuevos estándares para la acreditación de las carreras de Ingeniería establecidas por el Ministerio de Educación?

$\alpha$ ¿Sabías que durante el año 2018 la Facultad realizó una capacitación para la formación de formadores?

$\alpha \quad$ ¿Participaste de alguna capacitación? (particular, como referente, departamento)

$\alpha$ ¿Cómo te ves frente a este desafió de cambio?

$\alpha \quad ¿$ Cuánto se aleja tu práctica docente en relación a este modelo?

$\alpha \quad ¿$ Consideras que realizas prácticas docentes que se encuadran con el nuevo modelo?

$\alpha \quad ¿$ Cuáles se orientan a las competencias genéricas y cuáles a las específicas de la terminal?

$\alpha \quad$ ¿Estás participando activamente en la reformulación de los planes de estudio? ¿Qué opinas sobre este cambio de modelo educativo? 
Con el material resultante de las entrevistas se realiza un análisis cualitativo por categorías. Cabe aclarar que se toman todas las producciones de entrevistas como un texto único. Entonces, el análisis del texto representa un doble aporte: por una parte, se centra en la comprensión del contexto psico-social para profundizar cómo describen y comprenden esta transición y cómo les impacta en su vida laboral cotidiana.

A partir de sus resultados se implementa el análisis de contenido discursivo como técnica utilizada para profundizar en las variables emergentes que surgen de las entrevistas mencionadas y que conforman la muestra.

\subsubsection{Muestra}

La muestra es de carácter intencional, han sido seleccionados 9 docentes de distintos departamentos de la institución con dedicación exclusiva o parcial que participan en asignaturas de diferentes años de las carreras.

$\mathrm{Su}$ composición de género, sus profesiones, departamento de pertenencia y dedicación en el cargo universitario se muestra en la Tabla 1.

Tabla 1: Composición de la muestra

\begin{tabular}{|c|c|c|c|}
\hline Género & Carrera & Departamento & $\begin{array}{l}\text { ación } \\
\text { cargo }\end{array}$ \\
\hline $\begin{array}{l}4 \text { femeninos } \\
5 \text { masculinos }\end{array}$ & $\begin{array}{l}1 \text { Bioingeniero } \\
1 \text { Ingeniero Electrónico } \\
1 \text { Ingeniero en Alimentos } \\
2 \text { Ingenieros en Sistemas } \\
2 \text { Ingenieros Mecánicos } \\
2 \text { Ingenieros Químicos }\end{array}$ & $\begin{array}{l}3 \quad \text { Ingeniería Electrónica y er } \\
\text { Computación } \\
1 \text { Ingeniería Industrial } \\
1 \text { Ingeniería Informática } \\
1 \text { Ingeniería Mecánica } \\
1 \text { Matemática } \\
\text { 2 Ingeniería Oúmica v en Alimentos }\end{array}$ & $\begin{array}{l}7 \text { exclusivos } \\
2 \text { parciales }\end{array}$ \\
\hline
\end{tabular}

\subsection{Variables}

Para la recolección de datos se examinan los siguientes espacios de variable en estudio:

Socio-demográficas

a) Género

b) Profesión

c) Cargo docente

Percepciones de los docentes

a) Conoce/desconoce el proceso de cambio de modelo de enseñanza-aprendizaje basado en la formación por competencias.

b) Se interiorizó sobre los nuevos estándares

c) Realización de capacitaciones relacionadas al tema.

d) Percepción sobre el propio rol frente al cambio.

e) El gap entre las prácticas actuales y las que se encuadran con el nuevo modelo.

f) Participación activa en la reformulación de los planes de estudio. 


\subsection{Procedimiento: Análisis de los Datos}

Se analizan las variables sobre sus percepciones a través de una técnica específica, el análisis de contenido del discurso; por lo tanto, en primer lugar, se ordenaron y evaluaron sus percepciones en torno al tema para luego construir categorías emergentes de la presente muestra y su análisis posterior. La autora Vasilachis de Gialdino [21], considera a la investigación como un proceso interactivo entre el investigador y los participantes, como si se tratara de una técnica descriptiva y analítica, que privilegia las palabras de las personas y sus comportamientos observables como datos primarios. Por lo tanto, concluye diciendo que este modo de investigar es considerado como una forma de pensar, más que como una colección de estrategias técnicas.

El procedimiento metodológico supone, como se ha mencionado, con el material recogido la construcción de las categorías existentes para realizar sus respectivos análisis de contenido. Estas operaciones se dividen en tres procesos, que se consideran importantes e imposibles de abordar de modo unilateral. Por un lado, se trabajó con el análisis temático del material discursivo (contabilizar palabras según tópicos) [13], luego se procedió a realizar un análisis estructural del material discursivo (buscar su estructura interna: oposiciones y pares antitéticos) y finalmente un análisis situacional del material discursivo (denominado social-hermenéutico). Desde esa perspectiva, es decir, desde la pragmática del discurso, la dimensión hermenéutica implica la comprensión de relaciones e intereses concretos que los participantes perciben sobre sí o lecturas de otros sobre actores involucrados.

\subsubsection{Resultados: Análisis cualitativo}

A continuación, se presentan los resultados a partir de la técnica del análisis discursivo que implica la realización de un análisis cualitativo en tres aristas: a) análisis informacional; b) análisis estructural y c) análisis social-hermenéutico.

Las operaciones de análisis del discurso se dividen en tres procesos, que se consideran importantes e imposibles de abordar de modo unilateral. Por un lado se trabajó con el análisis temático del material discursivo del autor Ibáñez [22], además se procedió a realizar un análisis estructural del material discursivo, y finalmente un análisis situacional del material discursivo.

\section{Algunos hallazgos generales}

La etapa del proceso de actualización de los planes de estudio analizada corresponde a la fase de sensibilización acerca del modelo por competencias, su conceptualización y su futura aplicación. Con respecto a ello, los entrevistados expresaron en su totalidad estar en conocimiento del proceso. Algunos se informaron a través de la convocatoria institucional y la minoría mediante su participación ante el requerimiento de su respectiva red para proponer tanto las actividades reservadas del título como los descriptores específicos de competencias de la terminal. A partir de esta pregunta surgieron reflexiones como: "me interesó bastante porque siempre tuve un pensamiento crítico (...) de la otra forma de enseñanza, la tradicional".

Al seguir profundizando en su grado de conocimiento, cuando se consultó si sabía que se trataba de un modelo por competencias centrado en el estudiante respondieron afirmativamente en su totalidad. Sin embargo, se evidencia que las respuestas se enfocan en tres categorías. La primera en 
cuanto a su participación institucional, la segunda en la formación como docentes y la tercera en la necesidad de que el profesional adquiera competencias transversales demandadas por el sector productivo. Se destacan de sus dichos: "Se trata de mejorar las capacidades blandas que como ingenieros no hemos recibido (...) y que valora el sector productivo"; "En las reuniones de la red se discutía el modelo centrado en el estudiante y si era necesario o no ese cambio y su implementación"; y "Me interesa mucho poder capacitarme para estar a la altura de este nuevo paradigma".

En cuanto al conocimiento del contenido de los nuevos estándares propuestos por el "Libro Rojo" del CONFEDI se vislumbra que todos tienen un conocimiento básico y centrado en su especialidad. Se podría pensar que este saber surge de la participación en las redes o comisiones internas a la FI-UNMDP, circunscribiendo a la propia carrera perdiendo la visión global de la formación del ingeniero en un entorno interdisciplinario.

Al indagar en los entrevistados acerca de si estaban informados sobre la capacitación interna a cargo de un especialista en competencias que fue planificada para el año 2018 por las autoridades de la Unidad Académica para referentes en los departamentos, en su totalidad manifestaron estar al tanto. Sin embargo, solamente un tercio de la muestra asistió; algunos de los no convocados manifestaron que les hubiera gustado estar presentes; y a otros les hubiera gustado saberlo por vías departamentales. De aquellos que asistieron algunos indican que lo han replicado dentro del propio departamento.

Al consultar sobre su participación en alguna capacitación en forma particular, o propia del departamento, se nota una mayor dispersión. Indican participación en charlas, capacitaciones a partir de los referentes del departamento y reuniones de la red disciplinar. De los que no han participado en ninguna actividad uno indica conocer experiencias realizadas en otras universidades.

En definitiva, más allá de esta capacitación institucional, sólo una minoría de los docentes ha concurrido a otras capacitaciones.

Cuando se les pregunta cómo se ven frente a este desafió de cambio la mayoría expresa que está preparado y expectante a pesar que ello significa revisar la manera de pensar y enseñar respecto al modelo vigente. Sin embargo, algunos piensan que se deberá dar un cambio generacional "porque se identifica una resistencia dado que requiere un mayor esfuerzo por parte de la planta docente" o que "genera que nos replanteemos las prácticas docentes". Otros indican que: "es un desafío que debemos abordar sin duda alguna"; "no estoy segura de que los estudiantes estén preparados para este cambio y tener tanta autonomía"; y "me siento bien y estoy contento que se llegue a implementar y no quede en los papeles".

En referencia a cuánto se aleja su práctica docente en relación a este modelo la mayoría considera que algunos aspectos han sido incorporados tal vez sin la denominación formal correspondiente a una formación por competencias. Los comentarios más relevantes fueron: "no es un cambio del que estemos tan alejados, deberíamos hacer explícito lo que hacemos implícitamente"; "se vienen implementando hace varios años algunas herramientas de la formación por competencia"; "está muy relacionada, se invierte mucho tiempo en introducir capacidades blandas: desarrollo interpersonal, trabajo en equipo y comunicación"; "no estamos tan alejados, pero siento que depende del grupo de estudiantes también"; "lo ve más aplicable en asignaturas de los últimos años, donde el docente debe actuar como guía"; "siempre consideré al alumno como partícipe (...) y evalúo el proceso más allá del resultado de la instancia de evaluación"; y "tendría que hacer muchos cambios. Vengo de la vieja escuela que representa un modelo diferente".

En la percepción de considerar que realiza prácticas docentes que se encuadran con el nuevo modelo y de ellas cuáles se orientan a las competencias genéricas y cuáles a las específicas de la 
terminal, también existe dispersión. Si bien la mayoría expresa que, en cierta medida, algunos aportan a las genéricas y otros a las específicas, muy pocos a ambas. Expresan como acciones que se desarrollan: videos para presentar una idea o proyecto; problemas complejos relacionados a actividades profesionales y trabajos integradores o de campo realizados en grupo.

En referencia a su participación activa en la reformulación de los planes de estudio, más del 75\% indica que participa, algunos a través de una comisión interna del departamento, otros por intermedio de los miembros de esa comisión.

Respecto a este cambio de modelo educativo las respuestas son dispares de acuerdo a la experiencia o punto de vista. Mientras que para algunos existe motivación y lo consideran "un desafío personal y colectivo" o "una oportunidad de mejora que hay que aprovechar" para otros es "difícil de implementar" porque entienden que existe falta de compromiso de parte de los claustros docente y de estudiantes para el cambio que ello implica.

\section{Profundizando el análisis de su contenido}

a) Análisis Informacional

Se consideran los dichos de los participantes en cuanto a sus expectativas y percepciones como un texto único, sin importar quién lo enuncia. Primeramente, se han dividido sus producciones en percepciones con una connotación positiva, negativa o indiferente para la muestra. De este análisis surgen categorías específicas, el procedimiento sería similar a contabilizar las palabras-frase, con el objeto de separar y ordenar su contenido.

Las categorías emergentes (ver Figura 1) que aparecen en los entrevistados según el análisis del contenido del discurso son las siguientes:

Primeramente, se interroga a la muestra sobre su conocimiento/desconocimiento del cambio global e institucional que se atraviesa; responden de modo afirmativo: en su totalidad están al corriente del cambio, tienen diferencias en cuanto al tratamiento de profundidad del estado en cuestión, igualmente las respuestas han sido positivas.

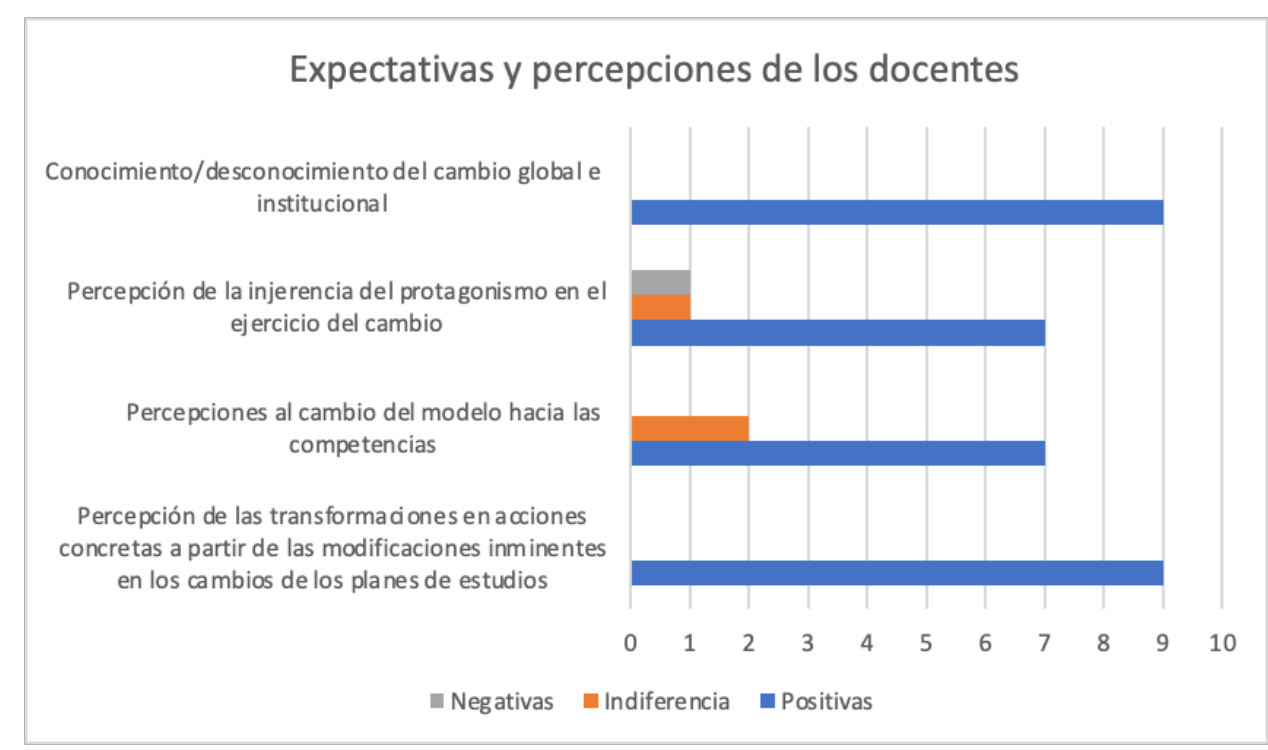

Figura 1: Expectativas y percepciones según el análisis del contenido del discurso. 
Percepciones con connotaciones que son positivas al cambio del modelo hacia las competencias (7), otras muestran cierta indiferencia (2) por desconocimiento, no se observa ninguna con connotación negativa. Lo interesante de este análisis informacional es que permite dar una visión más cuantitativa de los textos expresados por los participantes, para luego poder complementarlo con los otros análisis.

Percepción de la injerencia del protagonismo en el ejercicio del cambio positivas (7) dado que es integrante de comisiones al respecto, negativas (1) porque siente que no le dan lugar para participar en su departamento e indiferentes (1) porque no sabe cómo transmitir el entusiasmo a otros.

Percepción de las transformaciones en acciones concretas a partir de las modificaciones inminentes en los cambios de los planes de estudios positivas (9), dentro de las cuales existen matices dado que algunos lo consideran un desafío (3), otros lo relacionan con cambios organizacionales (2) y otros lo articulan a una gran motivación por el modelo (4).

b) Análisis estructural

Seguidamente se buscan establecer parámetros que den cuenta del funcionamiento interno del pensamiento de los integrantes de la muestra, es decir, poder establecer qué tipo de lógica organiza las ideas de los docentes. Por ejemplo, encontrar ideas-percepciones antinómicas que muestren oposiciones dentro de la población muestral sobre las categorías emergentes.

- Percepción de aceptación del modelo/ dificultad para práctica docente

Se establece una percepción común en el grupo que pone en evidencia la complejidad de llevar a la práctica el modelo: "Como docente, (...) es algo bastante complejo, hay que cambiar la forma de pensar y de enseñar". "Lograr más la participación y darle más importancia al estudiante como persona".

Otro participante señala "Estoy expectante, pero esto genera que nos replanteemos las prácticas docentes".

Alguien más agrega: "A mi todos los desafíos me gustan, lo que no me gustaría (como creo que le pasa a la mayoría de los docentes) que este desafio implique que se elimine mi asignatura o se le recorten los contenidos de forma impuesta".

Como se puede observar en ellos se hace evidente esta contradicción que la incertidumbre del propio cambio imprime, se quiere avanzar, pero se teme a perder la seguridad de lo conocido, lo ganado, el modo cómodo del ejercicio de su rol docente.

- Pensamientos positivos que imponen un ritmo

Se puede ver en la totalidad de los entrevistados, incluidos quienes sienten que al momento no han logrado participación o el modo de tener injerencia mayor en el cambio, que en su totalidad manifiestan una convicción que es el momento adecuado para movilizar la revisión de los planes de estudio y de las prácticas docentes en este sentido.

Si bien son bastantes críticos en cuanto a sus posibilidades y conocimientos sobre el modelo de enseñanza por competencias, tiene una visión positiva del mismo. 
c) Análisis social-hermenéutico

Para este tipo de estudio se tiene en cuenta el modo de comprender las ideas-percepciones de los participantes a la luz de su contexto social; es decir cómo explican ellos su pensamiento y qué cuestiones de su realidad se juegan para que resulten determinadas concepciones; en este caso puntual vinculadas a cómo piensan sus experiencias como docentes y qué impacto tienen estos cambios en su práctica diaria.

- Su práctica docente: impacto diario

Los participantes manifiestan mucha preocupación por mejorar su práctica docente, entendiendo que la implementación de este modelo trae consigo adaptaciones, revisiones y mucho esfuerzo personal para sostenerlo y adecuarlo en las aulas. Hay puntos coincidentes en sus explicaciones sobre cuáles serán los elementos a tener en cuenta:

- El acompañamiento desde los Departamentos de carrera: surgen temores a ciertas faltas de pensamientos comunes que formen una masa crítica.

- Un estudiante que se acople al cambio: conciben que quienes aprenden tiene una mayor participación en este modelo y ello solamente podría posibilitar el éxito en su ejecución.

- Reconocen una sensación de desafío personal implicado en este proceso lo cual los moviliza positivamente.

- Consideran que, si bien no trabajan actualmente desde el modelo de competencias, no lo ven muy alejado de su práctica docente. Algunos testimonios en referencia a este punto:

"hay cosas que hacemos pero no explicitamente, pero hacemos cosas que se relacionan a la formación por competencias"; "Considera que tal vez haga cosas que se encuadran pero que no lo hace conscientemente"; "no estamos tan alejados, pero siento que depende del grupo de estudiantes también"; "mi asignatura es totalmente práctica y es más importante evaluar las competencias que los conocimientos, por lo tanto mi actividad docente se ve afectada positivamente porque va a estar enmarcada en un plan que me permita evaluar por competencia"; "está muy relacionada, invierto mucho tiempo en introducir capacidades blandas: desarrollo interpersonal, trabajo en equipo y comunicación".

Tal como se puede leer en la propia voz de los participantes ellos dan explicaciones de cuáles serían los diferentes escenarios de aplicación del cambio hacia un modelo de formación que contempla competencias. Son conscientes de la importancia de cada integrante de la comunidad de la FI-UNMDP para poder llevar adelante el cambio, estudiantes, docentes, autoridades y sus voluntades alineadas solamente forjarán puentes que construyen este modelo singular con una lógica e identidad propia.

\section{Consideraciones finales}

Primeramente, se podría decir que en función de las expresiones vertidas por los entrevistados se resume que en relación al proceso de actualización de los planes de estudio están en conocimiento; así como también que ello, en sí mismo implica una mutación a un modelo por competencias.

Mayoritariamente la muestra estaba informada sobre el proceso de cambio, con un alto grado de participación tanto en sus departamentos académicos como en sus correspondientes redes 
disciplinares de terminales de carrera. Con respecto a su formación, su principal capacitaciòn fue la institucional.

Sin embargo, tienen percepciones positivas hacia la transición y en su mayoría expresan estar preparados y expectantes a pesar que ello significa una modificación profunda en el cambio de paradigma; dado que implica transformar el modo de pensar y enseñar respecto al modelo vigente. Algunos más conservadores en su visión consideran que habrá que esperar un cambio generacional para la renovación de la planta docente como única vía para que este cambio sea radical. Igualmente valoran que sus propias prácticas docentes no sean tan lejanas a este modelo.

Por otra parte, tienen consideraciones críticas en cuanto al aporte que realizan a competencias genéricas y/o específicas de cada terminal.

Por último, se puede concluir en que este trabajo se puede ver representada una parte de las voces de los docentes (con dedicaciones parciales y exclusivas), las cuales podría significar un insumo, sobre las percepciones de un grupo en referencia a la transición a hacia un modelo de formación por competencias. Como punto a destacar, la mayoría expresa sentirse que está preparado para asumir este desafío, lo cual es consecuente con sus acciones en sus prácticas docentes.

Finalmente, se considera que este proceso de transformación implica renuncias y movimientos, por tanto, cambios en ambos protagonistas: los docentes y los estudiantes.

\section{Referencias}

[1] E. Carterette y M. Friedman, "Manual de Percepción: Raices. Históricas y Filosóficas”. México D. F. : Editorial Trillas, 1982.

[2] C. A. Arias Castilla, "Enfoques teóricos sobre la percepción que tienen las personas". Horizonte Pedagógico. Volumen 8, No1 Año 2006 / p: 9 - 22

[3] CONFEDI Competencias Genéricas de Egreso del Ingeniero Argentino, CONFEDI, Buenos Aires, 2006.

[4] X. Roegiers, "Pedagogía de la integración: Competencias e integración de los conocimientos en la enseñanza". San José de Costa Rica. : Editorial Coordinación Educativa y Cultural Centroamericana, 2007.

[5] Comisión Europea Una introducción a Tuning Educational Structures in Europe. La contribución de las Universidades al proceso de Bolonia, Cimisión Europea, Bologna, 2006. [Online]. Disponible: http://www.unideusto.org/tuningeu/images/stories/documents/General_Brochure_Spanish_version.pdf Accessed on: Jun. 15, 2010

[6] P. Perrenoud, “Construir Competencias desde la Escuela”. Santiago de Chile. : Dolmen Ediciones, 2002.

[7] S. Tobón, J. Pimienta Prieto, J. García Fraile, "Secuencias Didácticas: Aprendizaje y Evaluación de Competencias”. México. : Pearson Educación. 2010.

[8] V. A.Kowalski, I. M. Erck, H. D. Enríquez, "Módulo 1: Competencias y Resultados de Aprendizaje". Programa de Formación Docente para orientar su práctica hacia la Formación por Competencias. Oberá, Misiones : Universidad Nacional de Misiones, 2018.

[9] U. Cukierman, “Aprendizaje Centrado en el Estudiante. Un enfoque imprescindible para la Educación en Ingeniería”. Buenos Aires. : CONFEDI. 2018.

[10] H. Perines y J. Murillo "Percepciones de los docentes en formación sobre la investigación educativa". Estudios Pedagógicos XLIII, N 1, 2017, pp. 251-268. 
[11] P. Perrenoud, "La Formación de los Docentes en el Siglo XXI", Revista de Tecnología Educativa, Santiago de Chile, XIV (3), 2001, pp. 503-523

[12] A. Giménez Giubbani, "El papel de la gestión de centros educativos en un modelo de aprendizaje basado en competencias", Revista Páginas de Educación, 9 (1), 2016 [Online]. Disponible: http://www.scielo.edu.uy/pdf/ pe/v9n1/v9n1a01.pdf

[13] A. Casado-Molina, y F. Cuadrado-Mendez, "La reputación corporativa: Un nuevo enfoque de las competencias transversales en el EEES”, Revista de Docencia Universitaria, 12, 2014, pp. 353-371

[14] E. Crisol Moya, "Reseña de Cómo hicieron los sistemas educativos con mejor desempeño del mundo para alcanzar sus objetivos. Barber, Michael y Mourshed, Mona" (2008), Revista de Currículum y Formación de Profesorado, vol. 14, núm. 2, 2010 [Online]. Disponible: https://goo.gl/CuxFgj).

[15] J.D. Morales Castillo, y M. Varela Ruiz, "El debate en torno al concepto de competencias", Investigación en Educación Médica, vol.4, n.13, 2015. [Online]. Disponible: http://www.scielo.org.mx/scielo.php? script $=$ sci_arttext\&pid=S200750572015000100007\&lng=es\&nrm=iso\&tlng=es

[16] P. Alonso-Martin, "La importancia y el nivel de desarrollo de las competencias en psicología", Psicología desde El Caribe, 25, 2010, pp. 84-107

[17] D. Clavijo, "El enfoque de competencias en la formación de abogados para el siglo XXI", Justicia, 27, 2015, pp. $185-2012$

[18] M. De Miguel, (Dir), I. Alfaro, P. Apodaca, J. Arias, E. García, C. Lobato y A. Pérez, "Modalidades de enseñanza centradas en el desarrollo de competencias". Orientaciones para promover el cambio en el espacio europeo de educación superior, España : Ediciones Universidad de Oviedo, 2005.

[19] A. Legault, “¿Una enseñanza universitaria basada en competencias? ¿Por qué? ¿Cómo?” Revista electrónica de desarrollo de competencias, REDEC, 5 (1), 2012.

[20] M. V. Artigas, A. E. Onaine, L. S. Santille; (2019, abril). "Haciendo foco en la conceptualización de las competencias: análisis del impacto para las carreras de Ingeniería". XI Congreso Argentino de Ingeniería Industrial COINI 2018. Memorias XI COINI 2018. [Online]. Disponible: https://ria.utn.edu.ar/xmlui/handle/20.500.12272/3501

[21] I. Vasilachis de Gialdino, "Estrategias de Investigación Cualitativa". Buenos Aires : Editorial Gedisa S.A., 2007.

[22] J. Ibañez, "Más allá de la Sociología. El grupo de discusión: técnicas y crítica". Madrid : Editorial Siglo XXI, 1979. 